\title{
Determining Projected Symmetries for Structural Fingerprinting of Nanocrystals
}

\section{Peter Moeck and Sergei Rouvimov}

Department of Physics, Portland State University, P.O. Box 751, Portland, OR 97207-0751

Because nanocrystals cannot be fingerprinted structurally from laboratory-based powder X-ray diffraction patterns, novel transmission electron microscopy (TEM) based structural fingerprinting strategies have been proposed [1]. These strategies utilize information on the projected reciprocal lattice geometry, 2D symmetry, and structure factors. While high resolution transmission electron microscopy images (HRTEM-Is) allow for the extraction of both structure factor amplitudes and phase angles of nanocrystals that are less than 5 to $10 \mathrm{~nm}$ thin, precession electron diffraction spot patterns (PED-SPs) allow for the extraction of structure factor amplitudes only [2]. For PED, the nanocrystals can be up to about five times thicker and the TEM's resolution is not limiting the structural fingerprinting [3]. A HRTEM-I and a PED-SP from the same crystal in the same orientation do, however, possess different 2D symmetries.

In order to facilitate the correct usage of 2D symmetry information for structural fingerprinting, we illustrate this effect for two crystals (with the same space group) in Figs. 1 and 2. In case of PEDSPs, a two-fold axis will be added to the plane group of the projected electrostatic potential and the 2D symmetry of the diffraction spot pattern will be that of the corresponding 2D point group. PEDSPs that show only reflections of the zero order Laue zone can, therefore, have only one of the 2D point groups: 2, 2mm, 4, 4mm, 6, or $6 \mathrm{~mm}$. This effect of the added two-fold axis is known as Friedel's law as applied to a plane section in reciprocal space. PED-SPs that were recorded with precession angles of a few degrees frequently show reflections from higher order Laue zones. Such reflections are not subject to the restriction of Friedel's law so that the 2D point groups 1, m, 3, and $3 \mathrm{~m}$ are also possible for the 2D symmetries of these reflections. In addition, large precession angles lead to a more effective integration of the reflection intensities so that small misorientations effects of the zone axis can be tolerated [3]. Fig. 1 shows a PED-SP (left) from a silicon crystal and compares it to an ordinary selected area electron diffraction spot pattern (SAED-SP) from the same area and with the same crystal orientation.

For sufficiently thin crystals and TEMs with sufficient resolution, the symmetry of the projected electrostatic potential, i.e. its 2D space group (which must be one of the 17 possible plane groups that exist in nature), is directly transferred to the HRTEM-Is. Figures 2 and 3 show HRTEM-Is from iron-oxide nanocrystals from a mixture of magnetite (space group: Fd3m) and cubic maghemite (space group: $\mathrm{P} 4{ }_{1} 32$ ). These two crystal structures are in the TEM undistinguishable on the basis of their projected reciprocal lattice geometry. Crystallographic Processing [4] of HRTEM-Is allows, however, for the determination of their plane groups and, thus, in certain projections of these nanocrystals for an unambiguous structural fingerprinting. Figure 3 illustrates this for a [001] oriented nanocrystal. While a magnetite crystal possesses plane group $\mathrm{p} 4 \mathrm{~m}$ in this projection, a maghemite crystal possesses the plane group p4g. Provided that the nanocrystals are sufficiently thin and possess not too many structural defects, one can distinguish between both plane groups reliably on the basis of the amplitude and phase residuals of the Fourier coefficients of the HRTEM-Is and the presence or absence of reflections that are kinematically forbidden due to the existence of glide lines in the latter plane group [5]. 


\section{References}

[1] P. Moeck and P. Fraundorf, Zeits. Kristallogr. 222 (2007) 634, open access:

http://www.atypon-link.com/OLD/toc/zkri/222/11?cookieSet=1 and arXiv:0706.2021

[2] P. Moeck and S. Rouvimov, In: Nano Particle Drug Delivery Systems: II Formulation and

Characterization, Y. Pathak and D. Thassu (eds.), Informa Health Care, New York, 2009, in press

[3] P. Moeck and S. Rouvimov, Zeits. Kristallogr. 2009, in press

[4] S. Hovmöller, Ultramicroscopy 41 (1992) 121.

[5] This research was supported by grants from the Oregon Nanoscience and Microtechnologies Institute and Portland State University's Venture Development Fund.
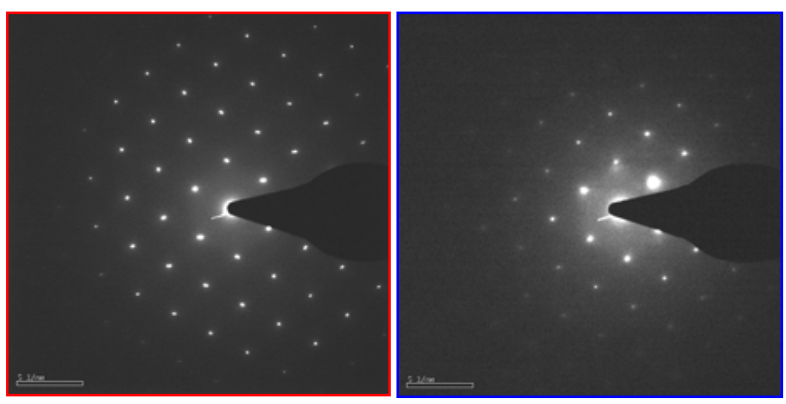

FIG. 1: PED-SP with $1.1^{\circ}$ precession angle (left) and SAED-SP (right) from the same area of an approximately $60 \mathrm{~nm}$ thick silicon crystals (space group $\mathrm{Fd} 3 \mathrm{~m}$ ), $200 \mathrm{kV}$, orientation close to [110]. While the 2D symmetry in the left spot pattern is $2 \mathrm{~mm}$, the right spot pattern has only the symmetry 2 due to its deviation from the zone axis orientation.
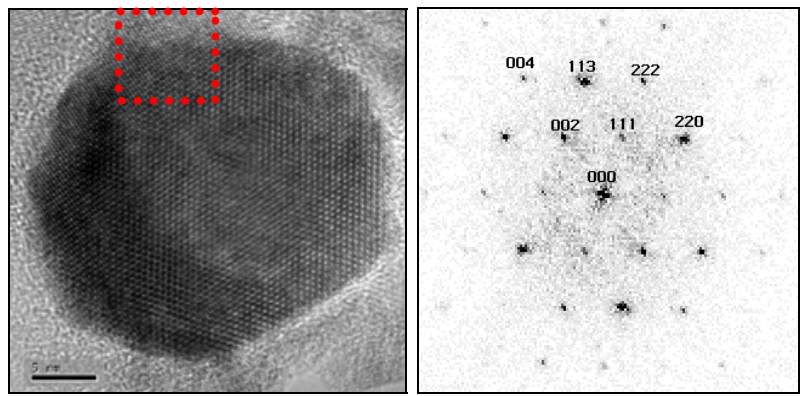

FIG. 2: HRTEM-I (left) and amplitude part of its (contrast inverted) Fourier transform (right), also known as digital diffractogram, of a magnetite nanocrystal, $200 \mathrm{kV},[110]$ orientation. The digital diffractogram was calculated for the marked area. While the plane group of the image area (left) is c $2 \mathrm{~mm}$, the $2 \mathrm{D}$ point group of its digital diffractogram (right) is $2 \mathrm{~mm}$.

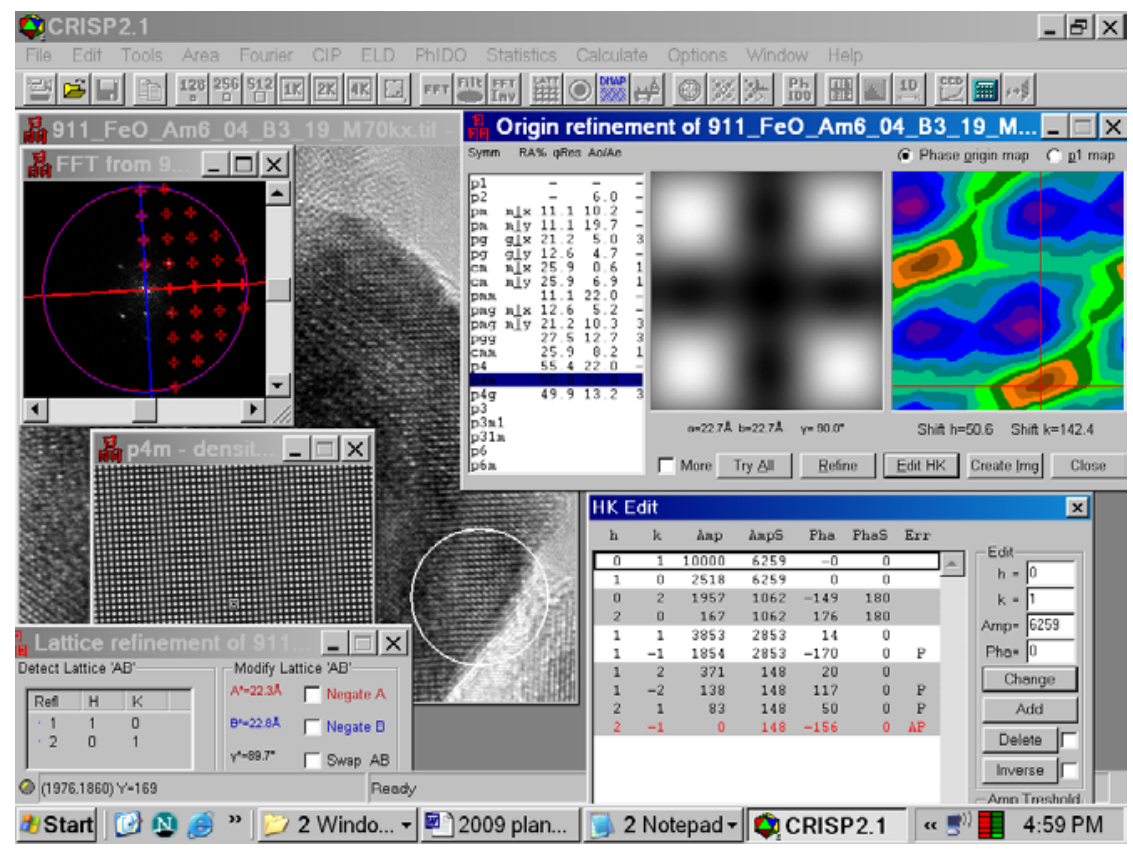

FIG 3: Crystallographic Image Processing [4] on some of the thinnest area of an ironoxide nanocrystal from a mixture of magnetite and maghemite. The relative low Fourier coefficient amplitude and phase residuals (for the highly symmetric plane groups $\mathrm{p} 4 \mathrm{~m}$ and $\mathrm{p} 4 \mathrm{~g}$ ) and the relative high intensity of plane group forbidden reflections $(\mathrm{h}=2 \mathrm{n}+1$ and $\mathrm{k}$ $=2 \mathrm{n}+1)$ of plane group $\mathrm{p} 4 \mathrm{~g}$ lead to an unambiguous identification of this nanocrystal as magnetite. 